\title{
Quantitating Glomerular Endothelial Fenestration: An Unbiased Stereological Approach
}

\author{
Behzad Najafian $^{a} \quad$ Michael Mauer $^{b}$ \\ ${ }^{a}$ Department of Pathology, University of Washington, Seattle, Wash., and ${ }^{\mathrm{b}}$ Departments of Pediatrics and \\ Medicine, University of Minnesota, Minneapolis, Minn., USA
}

\section{Key Words}

Contiguity $\cdot$ Endothelium $\cdot$ Fabry disease $\cdot$ Fabry

nephropathy $\cdot$ Fenestrae $\cdot$ Fenestration $\cdot$ Glomeruli •

Kidney $\cdot$ Morphometry $\cdot$ Stereology

\begin{abstract}
Background/Aims: Glomerular endothelial cells are fenestrated, allowing for especially high transcellular hydraulic conductivity. Current knowledge about endothelial fenestration structural changes in disease conditions is limited, partly due to the absence of robust methodologies to quantitate these structures. Herein, we propose a novel method for estimating the percentage of endothelial fenestration. Methods: An unbiased stereological method based on contiguity of two phases and surface area density estimation using isotropic uniform random line probes was developed. A line grid for intercept counting and classifying endothelial coverage of fenestrated versus non-fenestrated areas was designed. The method was applied to renal biopsies from 15 patients with Fabry disease and 9 normal living kidney donor controls. Results: The percentage of glomerular capillary endothelial coverage which was fenestrated was lower in Fabry patients ( $43 \pm 12 \%$ ) versus controls ( $53 \pm 9 \% ; p=0.047$ ). The fraction of endothelial surface which was fenestrated was greater on the peripheral versus mesangial zones of the capillary walls in both Fabry patients $(p=0.00002)$ and controls
\end{abstract}

$(p=0.0005)$. Conclusion: The proposed method provides an unbiased tool to quantitate endothelial fenestration changes in glomeruli. The practical example introduced showed reduced glomerular endothelial fenestration in Fabry nephropathy.

Copyright $\odot 2011$ S. Karger AG, Basel

\section{Introduction}

The hydraulic conductivity of glomerular capillary endothelial cells is especially high, allowing for a plasma filtration rate of about 180 l/day from human glomeruli. This is possible because of the unique properties of glomerular endothelial cells. In healthy glomeruli, most of the endothelial surface is extremely flat and contains many transcellular pores, or fenestrae $[1,2]$. The extent of endothelial fenestration may control the available glomerular basement membrane surface for plasma filtration. Thus, reduced glomerular filtration rate in preeclampsia has been attributed to loss of endothelial fenestration [3]. However, even in normal conditions, the endothelial cytoplasm is not uniformly fenestrated. The portion of the endothelial cell containing the nucleus is more frequently located on the mesangial side of the glomerular capillary wall and is usually non-fenestrated. However, a portion of mesangial capillary interface can 
be covered by flat fenestrated endothelium, while some of the peripheral capillary wall may be covered by non-fenestrated endothelium. Despite the probable physiologic importance of endothelial fenestration, little is known about how much these structures change in pathologic conditions. We recently reported reduced endothelial fenestration in diabetic nephropathy and Fabry nephropathy $[4,5]$, two conditions known to be associated with endothelial injury. Here, we describe the method that we used in those studies in more detail, including its theoretical background, sampling strategy, and application. In addition, we provide a practical example by applying the method to biopsies from patients with Fabry disease and healthy living kidney donor controls.

\section{Methods}

\section{Theoretical Background}

Contiguity is the degree of contact between two or more structures or phases. Here, we are interested in estimating the degree of contact between luminal capillary surface and its endothelial coverage which is fenestrated. This can be simply expressed as the ratio of fenestrated endothelial surface density to total capillary luminal surface density per a shared reference volume, such as glomerular tuft volume:

$$
C_{f c}=\frac{S_{V}(\text { FE } / \text { glom })}{S_{V}(\text { Cap } / \text { glom })}=S_{S}(\text { FE/Cap })
$$

where $C_{f c}$ is the contiguity between capillary luminal surface and fenestrated endothelium, $S_{v}(\mathrm{FE} / \mathrm{glom})$ is the surface area of fenestrated endothelial coverage of capillaries per glomerular volume, $S_{v}($ Cap/glom $)$ is the surface area of capillaries per glomerular volume, and $S_{s}(F E / C a p)$ is the surface area of fenestrated endothelium per total area of capillary endothelial coverage. Using an isotropic uniform random (IUR) grid of parallel lines, an unbiased estimator of the surface area per volume $\left(S_{v}\right)$ can be obtained [6]:

$$
S_{V}=2 \frac{\sum I}{\sum L}
$$

where $I$ is the line intercept count, and $L$ is the length of the line grid. If we use the same IUR grid for both phases (fenestrated endothelium and luminal capillary surface), equation 1 will be reduced to:

$$
\% E F=\frac{\sum I_{\text {fenestrated }}}{\sum I_{\text {total }}}
$$

where we have replaced $C_{f c}$ with the percentage of endothelial fenestration $(\% E F)$.

\section{Design of the Grid}

An unbiased counting frame $(224 \mathrm{~mm} \times 224 \mathrm{~mm})$ with fine parallel lines $4 \mathrm{~mm}$ apart with 1 coarse line per 7 fine lines was developed (fig. 1). Of note, the shape of an unbiased counting

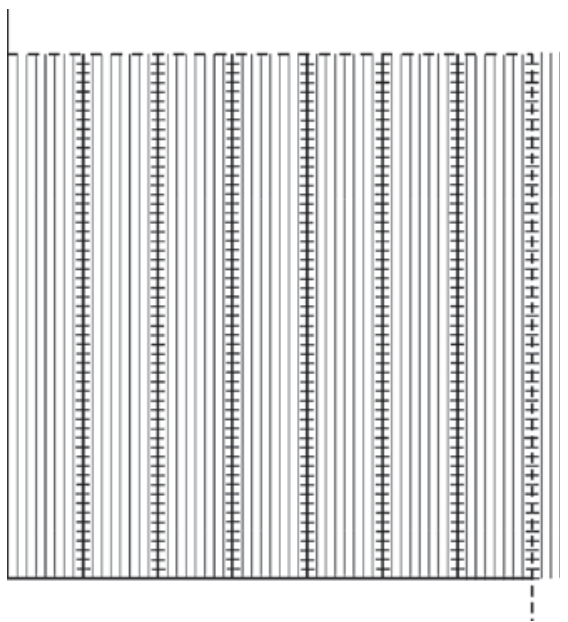

Fig. 1. The grid used to estimate $\% E F$ is composed of an unbiased counting frame with inclusion (dashed) and exclusion (continuous) borders, and parallel lines $4 \mathrm{~mm}$ apart. The shape of the counting frame was chosen based upon the use of the same grid for slit diaphragm length density (not required for endothelial fenestration studies). There is 1 coarse line (where the intercepts with endothelial coverage are counted) per 7 fine lines. The short lines on the coarse lines are $4 \mathrm{~mm}$ apart and, similar to fine lines, are used to define fenestrated versus non-fenestrated coverage. The endothelial coverage was arbitrarily called non-fenestrated if the distance between the two fenestrae on either side of the coarse line was more than 3 fine and coarse lines, otherwise it was called fenestrated.

frame was chosen since we use the same grid for filtration slit length density estimation (data not presented here). Moreover, this shape provides a guard zone around the frame to help recognize the structures approaching the frame borders. Figure 2 shows the grid superimposed on a glomerular capillary image. The intercept of each coarse line with capillary luminal surface was considered non-fenestrated if the distance between the two fenestrae on each side of the coarse line was more than 3 fine and coarse lines; otherwise it was called fenestrated (fig. 2). A section of endothelial cytoplasm is non-fenestrated according to our definition if the distance between margins of two adjacent fenestrae is $>230 \mathrm{~nm}$ [about 3 times the average diameter of a fenestrum in living kidney donor biopsies (data not presented)], at a magnification of about $\times 35,000$ (the magnification we use to study endothelial fenestration). The dashed lines on the coarse lines, similar to fine lines, are $4 \mathrm{~mm}$ apart and provide a guide to classify endothelial cytoplasm when the capillary wall is more parallel, rather than perpendicular to the grid lines.

\section{Biopsies and Sampling Protocol}

The study protocol was approved by the Institutional Review Board on Human Research at the University of Minnesota. Some of the patients with Fabry disease and all control subjects in this study were also included in our previous publication [4]. However, detailed endothelial fenestration data and methodologies were not previously presented. Biopsies from Fabry patients were obtained for clinical purposes, and informed consent was ob- 


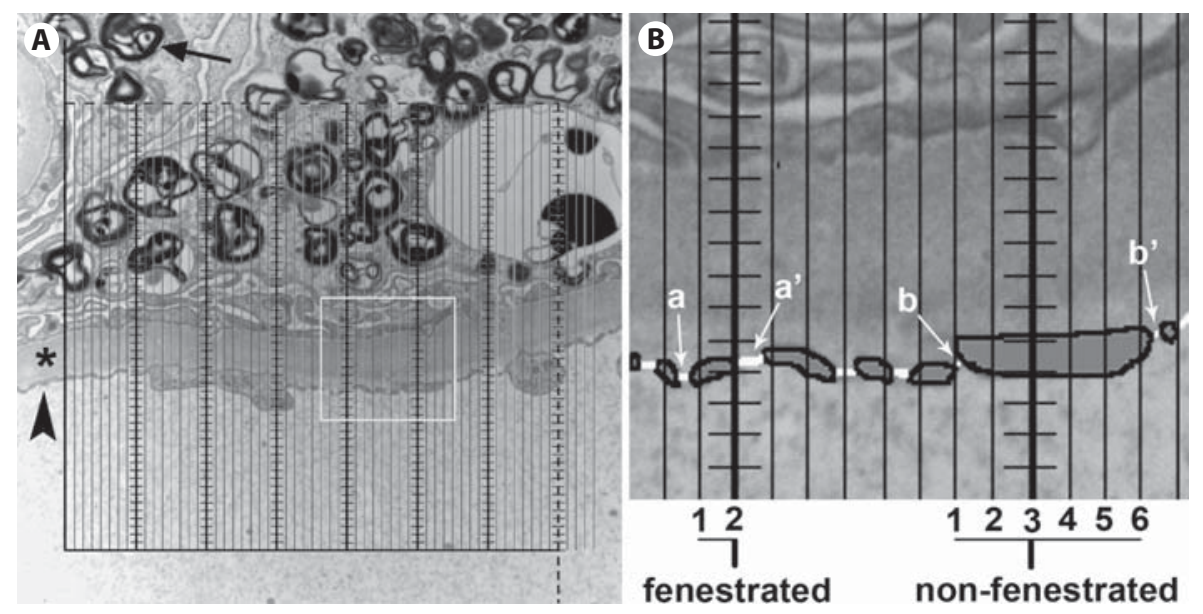

Fig. 2. A The grid in figure 1 is superimposed on a high magnification (about $\times 35,000$ ) of a glomerular capillary loop. The arrow marks a myelin figure inclusion (characteristic of Fabry disease) in a podocyte. The arrowhead points to capillary endothelial lining. The asterisk denotes the glomerular basement membrane. The white square shows the area magnified on the right. B Magnified view of a small portion of glomerular basement membrane with endothelial coverage on the bottom and podocyte foot pro-

tained to use these tissues for research. The living kidney donors had given their informed consent for research kidney biopsies to be obtained during transplant surgery.

Renal biopsies from 15 Fabry patients ( 10 males, 5 females, age $19 \pm 15$ years) and 9 living kidney donors (6 males, 3 females, age $30 \pm 13$ years) with normal renal function were processed for electron-microscopic studies. Toluidine blue-stained $1-\mu \mathrm{m}$ semithin sections were prepared to provide random profiles of glomeruli. The centermost glomerulus in each block with intact Bowman's capsule was selected on semithin sections. Ultrathin (60-90 nm) sections were mounted on Formvar-coated slot copper grids. Overlapping $\times 3,000$ images were obtained from the sampled glomeruli using a JEOL 100CX (JEOL, Tokyo, Japan) transmission electron microscope to prepare montages of the entire glomerular profile in Adobe Photoshop (CS3 extended). High-magnification images (about $\times 35,000$ ) were also obtained from each glomerulus according to a systematic uniform random sampling protocol (SURS). For this purpose, once a glomerulus at low magnification was identified, the position of the thin section grid was moved to the top (or bottom)-most portion of the glomerular tuft. Then, the grid was slightly moved horizontally to assume a 'random' start position adjacent to the capillary tuft. SURS was accomplished by moving the transmission electron microscope grid position control wheel at $120^{\circ}$ intervals horizontally and at $180^{\circ}$ intervals vertically, taking a picture at each stop point until the entire glomerular profile was scanned through (fig. 3).

A blinded observer screened montage images to exclude glomeruli with discernible mechanical or fixation artifacts. Highmagnification images were used for $\% E F$ estimation. Capillary walls were divided into peripheral and mesangial regions by drawing a line where glomerular basement membranes and capil- cesses on the top. The endothelial lining is traced with bold lines and shaded solid grey for easier visualization. Fenestrae are traced with white lines. Only intercepts of coarse lines (thicker with horizontal short lines) with endothelial lining are counted. The count of fine lines between closest fenestrae on either side of a coarse line $\left(a-a^{\prime}\right.$ and $b-b$ ') arbitrarily classifies the endothelial coverage as fenestrated versus non-fenestrated.

lary walls lost their parallelism. The grid shown in figure 1 was superimposed on these high-magnification images and intercepts between coarse lines and the endothelial cytoplasm in direct contact with the glomerular basement membrane were counted and classified as fenestrated or non-fenestrated as described above (fig. 2). \%EF was calculated using equation 3.

\section{Statistical Analysis}

Data are presented as means $\pm \mathrm{SD}$. Group comparisons were performed using unpaired two-tailed Student's t test or analysis of variance with least-square distance post hoc test. Linear regression analyses were performed for intervariable relationships. Normality of distributions was tested using Shapiro-Wilk test. Biological variation in the control and Fabry groups were separately estimated, considering that $C V^{2}$ total $=C V^{2}$ estimator + $C V^{2}$ biological variation, where $C V$ is the coefficient of variation. $\mathrm{p}$ values $<0.05$ were considered statistically significant.

\section{Results}

Two to five glomeruli (median $=3$ ) per biopsy were studied. On average, the number of intercepts between probe lines and capillary luminal surface was $295 \pm 107$ per biopsy, of which $217 \pm 83$ intercepts were on the peripheral and $78 \pm 37$ intercepts were on the mesangial zones. These accounted for $76 \pm 8$ and $72 \pm 7 \%$ of the capillary wall surface area to be peripheral (filtration zone) in Fabry patients and control subjects, respectively 
Fig. 3. Schema of systematic uniform random sampling of a glomerular profile simulated on a montage image $(\times 3,000)$ of a glomerulus from a Fabry patient. Asterisk marks a random starting point on the top left corner of the glomerulus. Squares simulate high-magnification images $(\times 35,000)$ obtained. Arrows show the pattern of moving the thin section grid using control wheels in a transmission electron microscope to uniformly sample the entire glomerular profile.

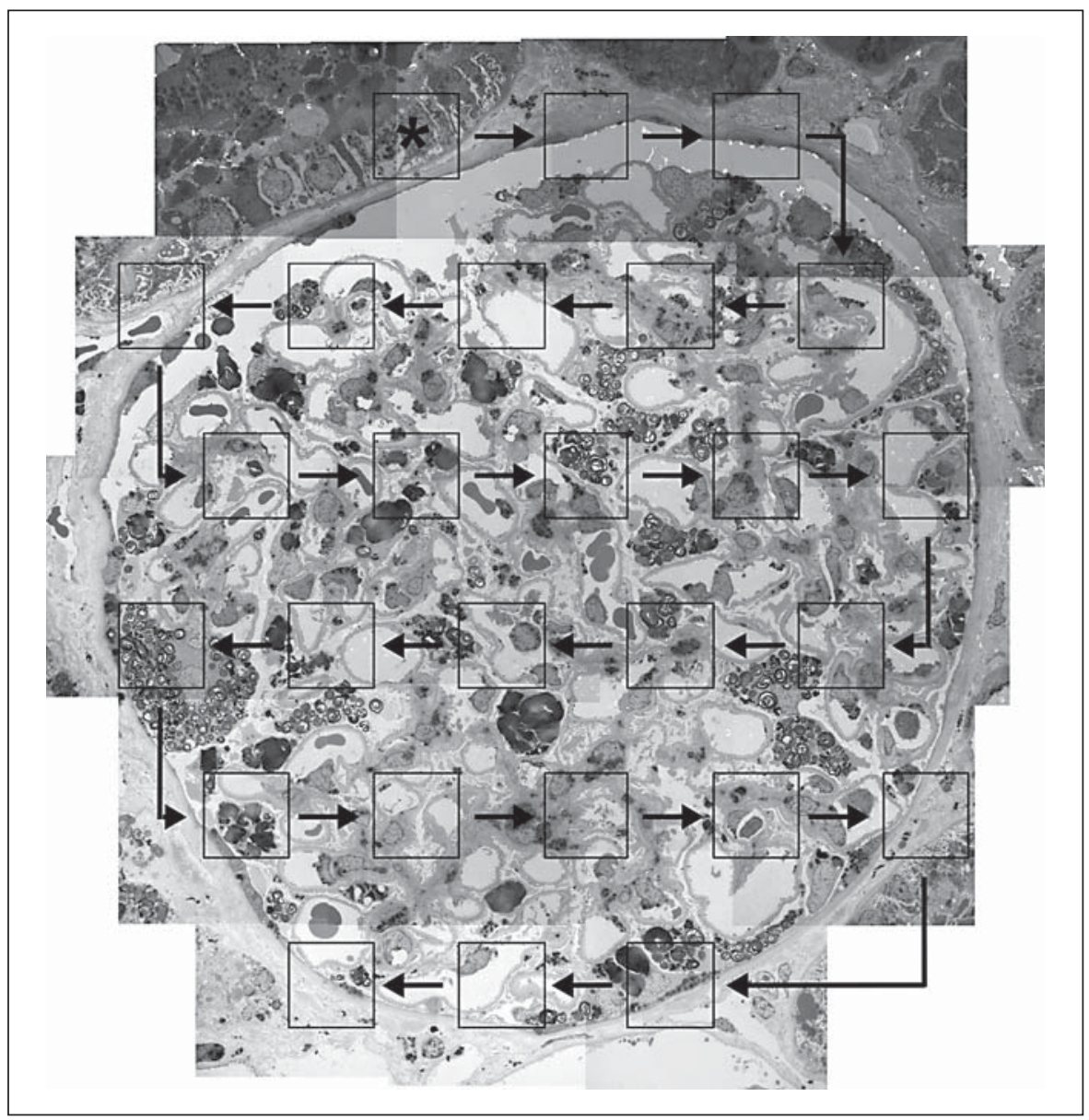

Table 1. $\% E F$ in glomerular capillaries in Fabry patients and control subjects

\begin{tabular}{|c|c|c|c|c|}
\hline & \multicolumn{3}{|c|}{ Fabry patients } & \multirow{2}{*}{$\begin{array}{l}\text { Controls } \\
\text { ( } 6 \text { males } \\
3 \text { females) }\end{array}$} \\
\hline & $\begin{array}{l}\text { males } \\
(n=10)\end{array}$ & $\begin{array}{l}\text { females } \\
(\mathrm{n}=5)\end{array}$ & $\begin{array}{l}\text { total } \\
(\mathrm{n}=15)\end{array}$ & \\
\hline \multicolumn{5}{|l|}{$\% E F$} \\
\hline Total & $43 \pm 14$ & $43 \pm 9$ & $43 \pm 12^{*}$ & $53 \pm 9$ \\
\hline Peripheral & $49 \pm 17$ & $48 \pm 9$ & $49 \pm 14^{* *}$ & $61 \pm 9$ \\
\hline Mesangial & $27 \pm 13$ & $19 \pm 5$ & $24 \pm 11$ & $33 \pm 17$ \\
\hline$\%$ peripheral & $74 \pm 8$ & $81 \pm 7^{*}$ & $76 \pm 8$ & $72 \pm 7$ \\
\hline
\end{tabular}

${ }^{*} \mathrm{p}=0.047$ vs. controls; ${ }^{* *} \mathrm{p}=0.04$ vs. controls.

[p = not significant (NS)]. \%EF in glomeruli followed a Gaussian distribution (Shapiro-Wilk test, $\mathrm{p}=\mathrm{NS}$ ) in both Fabry and control subjects. Table 1 presents the $\% E F$ measurements. On average, total (peripheral + mesangial) $\% E F$ was less in Fabry patients $(43 \pm 12 \%)$ versus control subjects (53 $\pm 9 \%$; $p=0.047$ ). Similarly, $\% E F$ on the peripheral zones of capillary walls was less in Fabry patients versus controls $(\mathrm{p}=0.04)$, while $\% E F$ on the mesangial zones was not statistically different between the groups. $\% E F$ was significantly greater in peripheral versus mesangial zones: $49 \pm 14$ versus $24 \pm 12 \%$ in Fabry patients $(\mathrm{p}=0.00002)$ and $61 \pm 9$ versus $33 \pm 17 \%$ in controls $(\mathrm{p}=0.0005)$. These two parameters were directly correlated in Fabry patients $(r=0.76, p=0.001)$, but not 
in the control subjects. There was no relationship between total, mesangial, or peripheral $\% E F$ and age, nor were these structural parameters different between males and females in the Fabry or control groups.

$C V$ of $\% E F$ in biopsies (interbiopsy variation) was $17.7 \%$, while the average $C V$ of the estimator (interglomerular variation) was $4.8 \%$ in the control group. These provided that the biological variation of $\% E F$ among the control subjects was about $17 \%$. Among the Fabry patients, interbiopsy variation of $\% E F$ was $28.5 \%$, the average $C V$ of the estimator was $12.3 \%$, and the biological variation among the patients was $25.7 \%$.

\section{Discussion}

The stereological method for estimating the fraction of capillary endothelial coverage which is fenestrated proposed here is based on the concept of contiguity between two phases, i.e. capillary internal surface and fenestrated endothelium. The ratio of a surface area to another surface area, as a measure of contiguity, was estimated using line probes [6]. Conceivably, since the estimated parameter is a dimensionless ratio, there is no need to correct for magnification. The prerequisite of the method is IUR sampling, meaning that the structure under study should have equal probability of being sampled by the probe at any direction in space. Because of the complex shape of the glomerular capillary network, branching frequently in almost any direction, a random section through a glomerulus provides multiple profiles of capillaries in various directions, mimicking IUR sampling from them. However, if the structure of interest is clearly anisotropic, i.e. vasa recta or loops of Henle, it would be advisable to implement methods to create isotropic sections, such as orientator [7], or alternatively prepare vertical sections followed by using cycloid grids for surface area estimation $[8,9]$. When the structure of interest needs to be studied at higher magnifications, sampling the entire structure may be tedious and unnecessary. SURS is an efficient way to obtain a representative unbiased sample of the structure [10]. Usually, a pilot study determines the sampling strategy [how many cases per group; how many glomeruli per biopsy; how many fields (images) per glomerulus, and how many lines per grid]. The goal is to minimize variation of the estimator (noise), while maximizing sampling efficiency, or in other words, to try to make the total variation (estimated from our measurements) as close to the biological variation as possible. In the present study, the coefficient of variation of estimator was less than 5\% in the control group and about $12 \%$ in Fabry patients, indicating that our sampling strategy adequately reduced the noise of estimation, while the difference between the two groups (mainly due to biological variation) was readily detectable. Biological variation among the Fabry patients was almost twice that of control subjects, reflecting greater heterogeneity of $\% E F$ in the Fabry patients.

Mechanisms controlling endothelial fenestration are not fully understood [11]. Vascular endothelial growth factor (VEGF) plays a crucial role in endothelial fenestration $[12,13]$. Podocytes are the main source of VEGF in glomeruli [14]. Glomerular endothelial cells express VEGF receptors (VEGFR) 2 and VEGFR3 [12, 15]. Reduced endothelial fenestration is a known finding in conditions with severe endothelial injury, such as preeclampsia and hemolytic uremic syndrome. In fact, reduced glomerular filtration rate in preeclampsia has been attributed to loss of endothelial fenestration [16]. Increased circulating levels of VEGFR1, which can bind to VEGF produced by podocytes, reduce the available VEGF to endothelial cells in preeclampsia, leading to loss of fenestration [17].

We previously reported reduced endothelial fenestration in human diabetic nephropathy, even in its very early stages $[4,5]$. Thus, endothelial fenestration was reduced in normo-albuminuric, micro-albuminuric, and proteinuric patients compared to control subjects [5]. Baelde et al. [18] reported reduced VEGF-A mRNA expression in biopsies from diabetic nephropathy patients which correlated with reduced expression of podocyte markers, suggesting a link between podocyte and endothelial injury.

Fabry is an X-linked hereditary disease with reduced or absent activity of the enzyme $\alpha$-galactosidase A, resulting in the accumulation of substrates, mainly globotriaosylceramide, in virtually all cell types in the body and in serious complications in multiple organs. Progressive renal failure is one of the major causes of morbidity in Fabry disease patients. The pathophysiologic mechanisms leading to reduced endothelial fenestration in Fabry nephropathy are unknown. Podocyte injury, an early finding in Fabry nephropathy, could potentially lead to reduced VEGF production [4]. In addition, Moore et al. [19] reported increased circulating soluble VEGFR2 in children with Fabry disease compared to control subjects. It is possible that, similar to preeclampsia, circulating VEGFR2 may reduce availability of podocyte-produced VEGF-A and VEGF-C to endothelial cells through increased binding to these molecules, thus leading to reduced fenestration.
Najafian/Mauer 
Also interesting was our finding of $33 \%$ fenestrated endothelial coverage on the mesangial zone of the capillary luminal surface. While it is likely that endothelial fenestration in the peripheral zone of capillaries is necessary to provide a high hydraulic conductivity to the glomerular filtration barrier, the role of fenestrae in the mesangial zone is not clear. However, it has been shown that mesangial cells actively participate in uptake and trafficking of plasma protein macromolecules [20]. Therefore, hypothetically, mesangial endothelial fenestrae may provide an alternative filtration path through the glomerular mesangium.

Current knowledge about the effect of various pathological conditions on endothelial fenestrae is scarce, perhaps, at least in part, due to the technical difficulties to study these structures, and, particularly, due to the previous absence of robust methodology to provide a quantitative measure of these structures. Applying the proposed methodology to biopsies from other renal diseases might improve our understanding of the contribution of structural changes of glomerular endothelial cells to changes in glomerular function and to the progression of glomerular injury.

\section{Acknowledgment}

We would like to thank Ann Palmer for stereological measurements and preparation of tissues for electron microscopy studies and Emily Glynn for preparing the glomerular montage shown in figure 3 .

\section{Disclosure Statement}

B.N. received a travel grant from Genzyme to present part of the data presented in this paper at the 'Focus on Fabry Nephropathy' satellite meeting of the World Congress of Nephrology 2009, Bergamo, Italy. M.M. is a member of the Genzyme-sponsored Fabry Registry Board, is a Genzyme consultant and has been a paid speaker at Genzyme-sponsored events. These studies were supported by NIH grant 5U54NS065768, an investigator-sponsored research award (Natural history and structural functional relationships in Fabry renal disease) from Genzyme, and a Grantin-Aid of Research, Artistry and Scholarship from the Graduate School of the University of Minnesota.

\section{References}

-1 Farquhar MG, Vernier RL, Good RA: An electron microscope study of the glomerulus in nephrosis, glomerulonephritis, and lupus erythematosus. J Exp Med 1957;106:649660.

2 Kondo $\mathrm{H}$, Ushiki T: Stratified laminae fenestratae (alveolus fenestratus endothelialis) in the glomerular capillaries of the mouse kidney. Arch Histol Jpn 1985;48:117-122.

3 Avasthi PS, Evan AP, Huser JW, Luft FC: Effect of gentamicin on glomerular ultrastructure. J Lab Clin Med 1981;98:444-454.

4 Najafian B, Svarstad E, Bostad L, Gubler MC, Tondel C, Whitley C, Mauer M: Progressive podocyte injury and globotriaosylceramide (GL-3) accumulation in young patients with Fabry disease. Kidney Int 2011; 79:663-670.

5 Toyoda M, Najafian B, Kim Y, Caramori ML, Mauer M: Podocyte detachment and reduced glomerular capillary endothelial fenestration in human type 1 diabetic nephropathy. Diabetes 2007;56:2155-2160.

6 Weibel ER: Stereological Methods: Practical Methods for Biological Morphometry. London, Academic Press, 1979.

7 Mattfeldt T, Mall G, Gharehbaghi H, Moller $P$ : Estimation of surface area and length with the orientator. J Microsc 1990;159:301-317.

8 Gokhale AM, Benes VV: Estimation of average particle size from vertical projections. J Microsc 1998;191:195-200.
9 Gokhale AM, Evans RA, Mackes JL, Mouton PR: Design-based estimation of surface area in thick tissue sections of arbitrary orientation using virtual cycloids. J Microsc 2004; 216:25-31.

10 Gundersen HJ, Jensen EB: The efficiency of systematic sampling in stereology and its prediction. J Microsc 1987;147:229-263.

11 Satchell SC, Braet F: Glomerular endothelial cell fenestrations: an integral component of the glomerular filtration barrier. Am J Physiol Renal Physiol 2009;296:F947-F956.

12 Kamba T, Tam BY, Hashizume H, Haskell A, Sennino B, Mancuso MR, Norberg SM, O'Brien SM, Davis RB, Gowen LC, Anderson KD, Thurston G, Joho S, Springer ML, Kuo CJ, McDonald DM: VEGF-dependent plasticity of fenestrated capillaries in the normal adult microvasculature. Am J Physiol Heart Circ Physiol 2006;290:H560-H576.

13 Sugimoto H, Hamano Y, Charytan D, Cosgrove D, Kieran M, Sudhakar A, Kalluri R: Neutralization of circulating vascular endothelial growth factor (VEGF) by anti-VEGF antibodies and soluble VEGF receptor 1 (SFLT-1) induces proteinuria. J Biol Chem 2003;278:12605-12608.

14 Foster RR, Satchell SC, Seckley J, Emmett MS, Joory K, Xing CY, Saleem MA, Mathieson PW, Bates DO, Harper SJ: VEGF-C promotes survival in podocytes. Am J Physiol Renal Physiol 2006;291:F196-F207.
15 Foster RR, Slater SC, Seckley J, Kerjaschki D, Bates DO, Mathieson PW, Satchell SC: Vascular endothelial growth factor- $\mathrm{C}$, a potential paracrine regulator of glomerular permeability, increases glomerular endothelial cell monolayer integrity and intracellular calcium. Am J Pathol 2008;173:938-948.

16 Lafayette RA, Druzin M, Sibley R, Derby G, Malik T, Huie P, Polhemus C, Deen WM, Myers BD: Nature of glomerular dysfunction in pre-eclampsia. Kidney Int 1998;54:1240-1249.

17 Stillman IE, Karumanchi SA: The glomerular injury of preeclampsia. J Am Soc Nephrol 2007;18:2281-2284.

18 Baelde HJ, Eikmans M, Lappin DW, Doran PP, Hohenadel D, Brinkkoetter PT, van der Woude FJ, Waldherr R, Rabelink TJ, de Heer E, Bruijn JA: Reduction of VEGF-A and CTGF expression in diabetic nephropathy is associated with podocyte loss. Kidney Int 2007;71:637-645.

19 Moore DF, Krokhin OV, Beavis RC, Ries M, Robinson C, Goldin E, Brady RO, Wilkins JA, Schiffmann R: Proteomics of specific treatment-related alterations in Fabry disease: a strategy to identify biological abnormalities. Proc Natl Acad Sci USA 2007;104: 2873-2878

20 Mauer SM, Fish AJ, Blau EB, Michael AF: The glomerular mesangium. I. Kinetic studies of macromolecular uptake in normal and nephrotic rats. J Clin Invest 1972;51:10921101. 